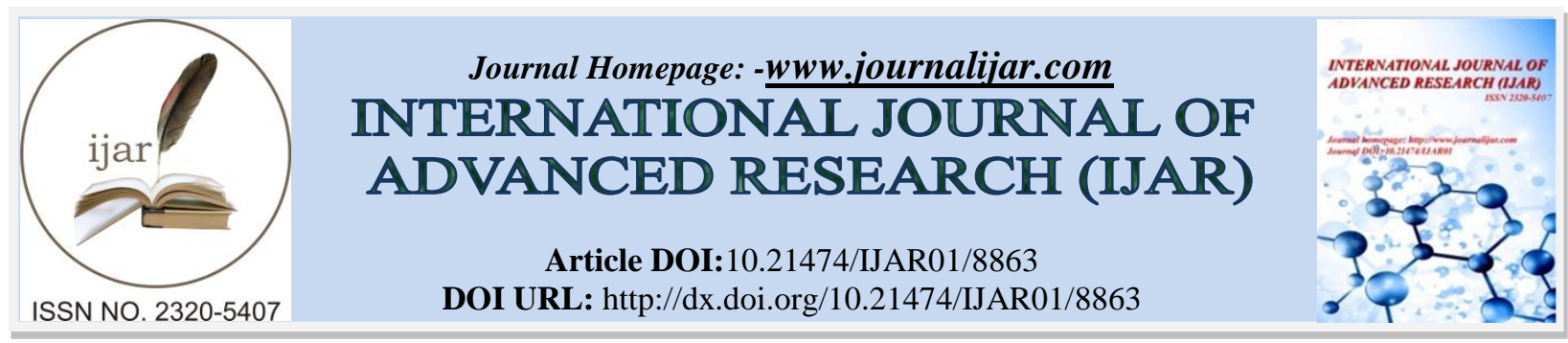

RESEARCH ARTICLE

\title{
ORIGIN, EXPANSION AND ABOLITION OF NARAYANI COIN OF THE PRINCELY STATE OF COOCH BEHAR.
}

Md. Jahirul Haque.

Department of History,Cooch Behar Panchanan Barma University,Cooch Behar, West Bengal, India.

\section{Manuscript Info}

Manuscript History

Received: 10 February 2019

Final Accepted: 12 March 2019

Published: April 2019

Key words:-

Indigenous,Medieval,Ascended,Narayan

i, Sovereignty,Devanagari etc.
Abstract

The Cooch Behar State was one of the mighty indigenous of India. The state had immense importance in ancient and medieval history of India. The Maharaja of Cooch Behar ruled the state for hundred years. The former Cooch Behar State has become a district of West Bengal since 1950. Maharaja Biswa Singha was the founder of the Cooch dynasty in Cooch Behar. After the death of Maharaja Biswa Singha, his son and successor Naranarayan ascended on the throne of Cooch Behat State in 1555 A.D. It became a vast empire during the reign of Maharaja Naranarayan. He was the most powerful king among all other kings of Cooch Behar State.The introduction of 'Narayani Coin' is one of the most important achievement of Maharaja Naranarayan which carries the identity of his sovereignty. It is unknown whether Maharaja Biswa Singha introduced coin in his name. No coin of Maharaja Biswa Singha has been discovered till date. The coin of the Cooch Behar State was first introduced in 1555 at the time of coronation of Maharaja Naranarayan.The coin is known as 'Narayani Coin'.Two types of coin, gold and silver, named after Maharaja Naranarayan were made.On the one side the name of the Devanagari character Mahadev was printed and the name of the king was printed on the other side. Narayani coins were prevailing in some other neighboring states including Cooch Behar and Assam. In the past,trade routes extenden from Magadha to China's Yunan through Assam.At that time the Narayani coin was the medium of trade in Jaintia, Khasi, Kachher, Manipur, Tripura with North East Asia. From the wide spread expansion of Narayani coin, it is understood that there was a great deal of trade and commerce in the state of Cooch Behar. In 1866, the British government of India stopped the circulation of Narayani coin. However, later on, the Maharaja of Cooch Behar used to prepare coin as a commemorative coin for the first day of ascendency.

Copy Right, IJAR, 2019,. All rights reserved.

\section{Introduction:-}

It is difficult to know the exact time when the coin was introduced in ancient Pragjyotish or Kamrupa. The coins of the Kushanas and the Gupta Kings were discovered in different parts of Bengal. After the Gupta Era the Palas and the Senas established their supremacy on Kamrupa but their coins have not been discovered. The coins of Giasuddin Euaz are the oldest than other Muslim rulers in Bengal (1210 cm 1222 A.D. $)^{1}$. Later on the coins of Samsuddin Ilias Shah along with other coins have been discovered. In 1863 some coins were discovered towards the south-eastern side on the bank of the river Dharla. Some of them belonged to the Pathan rulers of Gour and Delhi. The coins of Sikander Shah have also been discovered. In different parts of Bengal, the coins of Hossain Shah, ruler of Gauda have been discovered. But the coins of Nilamber and his former kings had not been discovered.

Corresponding Author:-Md. Jahirul Haque.

Address:-Department of History,Cooch Behar Panchanan Barma University,Cooch Behar, West Bengal, India. 
It is unknown to us about the introduction of coins of Maharaja Biswa Singha. The Daranga Vanshabali or Rajophakhyana left us no reference about it. No coin of Maharaja Biswa Singha has been discovered till date.Roy Gunaviram Barua mentioned in 'Assam Burunji' that the Cooch Kingdom had no coin before Maharaja Biswa Singha. ${ }^{2}$ After the accession on the throne, Maharaja Biswa Singha printed 'Sikka Rupee'. ${ }^{3}$ But none of these have discovered till now.

From the reign of Maharaja Nara Narayana, son of Maharaja Biswa Singha, the Cooch kings began to introduce their coins independently.Maharaja Nara Narayan introduced coin for the first time on the day of his coronation in 1555 A.D. ${ }^{4}$ On the one hand the name of the Devanagari character Mahadev was printed on the one side and the name of the king was printed on the other side. ${ }^{5}$ The coin of Cooch kingdom are known as Narayani coin. This name came after the name of King Nara Narayana. For this title the dynasty of Cooch kings is known as Narayani Dynasty. There is another reason about the naming of the Narayani coins. The name Narayani came after the name of Narayan, the Royal Deity of the Cooch kings. The Narayani coins were mentioned in Alamgirnama and Riaz-Us-Salatin. The images of the Siva or Siva-Durga were engraved in the coins. That is why these are called 'Sivatanka' or 'Sivankatanka'.

We have already known about the golden, silver, brass and copper coins of the Cooch kings. But the coins made of brass have not yet been discovered. The round shaped and introduction of inscription are the main characteristic of the Narayani coin. The title of the king was engraved on the obverse side and the name and date on the reverse side of the coin. This custom was followed up to the reign of Laxminarayana. Later on, the kings lost their authority in the making of their coin. Then they had only the right to make half-rupee.

From the reign of Maharaja Naranarayan, the Maharaja of Cooch Behar made their coins with silver and followed the custom of 'Tanka' belonging to the Muslim Sultan of Bengal. These coins were circular in shape. The impact of Muslim 'Tanka' is noticed on these coins and even these coins have special sign (Monogram and Klinkfoil) also. These signs marked under the word ' $N$ ' .

King RaghudevNarayana and his son Pariksheet Narayana made their coins in their own name.The writings of their coins read as follows:

"Sri Sri Haragaury

Charan Kamal Madhu

- karasya',.

The coins of Raghudev Narayana were 161.3 gren in weight. These are all made in imitation of the'Narayani coins'.

Maharaja Laxminarayana made his coins in his own name. The writing of his coins looked like the coins of king Nara Narayana.

The writting of the coin read as follows:

In one side:

Sri Sri Ma

Laxmi nara

Yana saka

1509.

On the other side:

Sri Sri Sivachran

Kamal Madhukarasya

A large number of coins belonged to Maharaja Pran Narayana have been discovered. Of these some are preserved in Cooch Behar Treasury, two in in Cooch Behar palace and one in Cooch Behar Sahitya Sabha. The rupees are 142154.3 gren and the half-rupees are 61.3 - 78.7 gren in weight. In these coins the references of 'Sake' which means 'Saka Era' or Rajasaka have been found. The following date 1554 saka, 1555 saka and 1559 saka have been found.

After Maharaja Pran Narayana only half-rupee of the later kings have been discovered. One half-rupee belonged to Maharaja Modnarayana of 179 Rajasake had been found. It is 75.28 gren in weight. One half-rupee belonged to Maharaja Basudev Narayana is now in Cooch Behar Treasury. King Rup Narayana's half-rupee have been found of which two in Cooch Behar Palace and others are in Cooch BeharTreasury.The coins of Maharaja Upendra 
Narayana, DebendraNarayana and Dhairjendra Narayana are preserved in Cooch Behar Palace, Treasury and Thakurbary. The coins of Maharaja Dharendra Narayana have not yet been discovered.

From Maharaja Shivendra Narayana, the introduction of modern Bengali letter on the front side was introduced. Sixteen gold coins, eight silver coins belonged to Maharaja Shivendra Narayana have been discovered. Of these eleven gold coins are preserved in treasury and other five in Thakurbari. Two coins made of silver are now in the existing Palace of Cooch Behar and some others in treasury.

Among the coins of king Narendra Narayana nine gold-coins are now in treasury, three in Thakurbari and half-rupee made of silver are in Thakurbari and the Palace. The coins of king Nripandra Narayana have also been discovered of which five made of gold are preserved in treasury, one in Thakurbari, among the half-rupee made of silver two are in Cooch Behar Palace, and over one thousand are in treasury. The coins of Maharaja RajrajendraNarayana made of gold and silver are pre - served in treasury.

The coins of Maharaja RajrajendraNarayana are totally different from the former kings of Cooch Behar. In his coins the traditional writings Sivacharan Kamalmadhukarasy" changed and "Jatodharmostato joyo" and the 'Coat of arms' (Lion and Elephant Image) are engraved. ${ }^{6}$. Maharaja Nara Narayana introduce, the Seal in which the figures of lion are engraved and it is called 'lion-seal'.

From 1929 the introduction la of tiger-figure in place of lion had been started. The coins of Maharaja RajrajendraNarayana, Jitendra Narayana and Jagaddipendra Narayana were made in Raj sake 402, 403 and 413. From 1778 the Narayani coins were divided into two groups - (A) Old Narayani Coin and (B) New Narayani Coin. The coins which were made from Maharaja Naranarayana to DebendraNarayana are called old Narayani coin and the coins made in later period were called new Narayani coin.

From 1773, the East India Company tried to curb the power of coin making of the Cooch Kings. In 1886, the British Government compelled the Cooch King to stop the making and using of coin. Then the British government introduced their own coin.

Narayani coin became popular and had heavy demand during the reign of Maharaja Nara Narayana. These Narayani coins were used all over Narth-Bengal, Nepal, Bhutan, Sikkim, Tibet and Assam. The coins of Jayantia look like the 'Narayani coins'.At that time the Narayani Coinwas the medium of trade in Jaintia, Khasi, Kachher, Manipur,Tripura with North East Asia. The Bhutan kings also made their coins in the mint of Cooch Behar.

There are four different marks in the Narayani coin. These marks are as follows: Dot marks $\left(^{\circ}\right)$, cross-mark $(\mathrm{X})$, half-moon-mark $\left(^{\wedge}\right)$ and combine mark of half moon and cross mark (\&). The half-moon only appears in the coins of Dharendra Narayanaa or Harendra Narayana and no mark was placed on the coins of the later rulers. There is great similarity between the sign of Narayani coins and some Buddhist Coins. The Cooch kingdom had a mint at Takagachh to the north-west of Cooch Behar town. The village named after the mint. This mint made 50 thousand coins in each year. A Royal Officer look xafter the work of the mint who was called as 'Bhander Thakur. ${ }^{7}$

Bharatiya Mudra Parished had published a journal in which coin specialist Sri Basanta Chaudhury and Sri Parimal Roy wrote an Essay about the coin made of gold of Maharaja Nara Narayanan. ${ }^{8}$. From the newly discovered coins it has been known that Maharaja Nara Narayana had struck it to make his victory memorable. This gold coin is 12.15 gren in weight and 2.98 millimeter in size. It has images of a lion in one side and the mark of half-moon alone with dot engraved by the side of it. The other side of the coin has an inscription which read as follows:

Sri SriHaragauri

Charan Kamala

Madhukarasya .

In1995 some Narayani coins were discovered at Chandirjhar of Alipurduar .A coin repository was discovered at Siddeshwari village of Cooch Behar.These coins were belong to the reign of Maharaja Naranarayan. ${ }^{9}$ In 2010 some silver coins were discovered under the ground at Dalimpur in Jalpaiguri district.These coins are belong to the reign of Maharaja Laxminarayan.The Narayani coins are still being discovered in several parts of Cooch Behar and its neighboring areas. 


\section{Conclusion:-}

The British Indian Government stopped the circulation of Narayani coin in 1866A.D. However, later on the Maharaja of Cooch Behar used to prepare coin as a commemorative coin for the first day on ascendency. ${ }^{10}$ Finally, the Princely State of Cooch Behar was merged with Indian Union in 1949. As a result, there was no need to prepare and circulation of coin for Cooch Behar. Today, the Narayani coins are treated as ancient archaeological evidence. There is a lot of research going on in the country and abroad with Narayani coin.

\section{References:-}

1. Khan Chowdhuri Amanatulla Ahmed:A History of Cooch Behar(In Bengali),printed at the State Press and published under Authority of the Cooch Behar State,1936,Reprint-1990,Kolkata,page-279.

2. Ranajit Deb: Cooch Beharer Rajprasad, Kolkata-6,2014, page-82.

3. Mukhopadhaya Shaymchand: Cooch Jelar Purakirty, Calcutta-9, 1974, page-63.

4. Op. Cit-Khan Chowdhuri Amanatulla Ahmed, page-101.

5. Munshi Jaynath: Rajopakhyan, Edited by Biswa Nath, Calcutta-36,1989, page-19.

6. Bhattacharya Asoke Kumar: Indian Numismatics And Its Cultural Aspects, Delhi,2010, page-112.

7. Roy Barma Hemanta Kumar: History of Cooch Behar (In Bengali), Printed at the Sulekha Printing Press of Cooch Behar,1988,page-198.

8. Das Biswa Nath: Cooch Behar Jelar Purakirti Samgraha, Calcutta-73,1985, page-64.

9. Basu Sankar Kumar: The Commercial Coinage of Cooch Kingdom, Library of Numismatic Studies, Kolkata64,2013, page-17.

10. Mallik Parthaprotim(Ed.): Srijani Dhara,Journal of Literature and Culture, Jalpaiguri,2018,page-160. 